Revista de Psicología de la PUCP. Vol. XV, 2, 1997.

\title{
ACERCA DE LA CONTRATRANSFERENCIA: ¿OBSTÁCULO O INSTRUMENTO?'
}

\author{
Jozef Corveleyn ${ }^{2}$ \\ Universidad Católica de Lovaina
}

[1 artículo discute las distintas perspectivas que dentro del mismo psicoanálisis existen sobre el estatuto de la contratransferencia en el proceso terapéutico. Mientras que para unos (la tendencia clásica) ésta debe ser radicalmente reprimida para salvaguardar el ideal de una neutralidad aséptica, para otros (la tendencia bumanista) ésta puede ser incorporad a para consolidar los aspectos externos y reales de la alianza terapéutica. Junto con la postura clásica de Theodor Reik y la más. moderna de Harold Searles, el autor plantea que los sentimientos y reacciones contratransferenciales pueden ser usados como un instrumento legitimo que oriente tanto la telación con el paciente como la labor interpretativa.

Palabras claves: técnica psicoanalítica, transferencia, contratransferencia.

\begin{abstract}
About countertransference: ¿Obstacle or tool?
This paper discusses the different perspectives that exist in psychoanalysis about the status of the countertransference in the therapeutic process. On the one hand, for those who follow the classical tendency this should be radically repressed to protect the ideal of an aseptic neutrality. On the other hand, for those who follow the humanistic tendency, this should be incorporated to consolidate the external and real aspects of the therapeutic alliance. Together with the classical work of Theodor Reik and the modern work of Harold Searles, the author proposes that countertransference feelings and reactions could be used as legitime tools to monitor both the relationship with the patient, as well as the interpretation phase.
\end{abstract}

Keywords: psychoanalitic technique, transference, countertransference.

1. El autor agradece a Jill de Ridder de la Universidad Católica de Lovaina por la traducción del artículo, y a César Pezo y Octavio Zagazeta de la Pontificia Universidad Católica del Perú, por la adaptación del mismo. las referencias a los textos de Freud se han cotejado con la versión castellana de $\Lambda$ morrortu Editores.

2. Profesor Principal de la Universidad Católica de Lovaina (Katholieke Universiteit Leuven), Bélgica. Es Licenciado en Filosofia, Licenciado y Doctor en Psicología y Psicoanalísta, miembro de la Escuela Belga de Psicoanálisis. Director del Centro de Invesitgaciones Psicoanaliticas del Departamento de Psicologin de la Facultad y de Psicología y Ciencias de la liducación de la Universidad Católica de Lovaina. Es, además, Profesor Honorario de la Universidad de Lima y coordinador general del Convenio de Cooperación Académica Universidad Católica de Lovaina - Universidad de Lima. Tiensestraat 102, B-3000, Leuven, Belgium; correo electrónico: Jozef. Corveleyn@psy.kuleuven.ac.be 

Bajo el concepto de contratransferencia y de manera indirecta también bajo el de transferencia se lee en la literatura analítica clásica sobre la importancia de la persona del analista y su implicancia personal en la terapia. En este texto seguimos algunas huellas del pensamiento acerca de este tema y hacemos algunas anotaciones precisando el rol de la contribución personal del terapeuta en el proceso terapéutico.

La definición del término contratransferencia es relativamente simple. Se refiere a los sentimientos y representaciones conscientes e inconscientes que son despertadas en el analista, en y por causa de la cercanía que produce, el escuchar al paciente. La contratransferencia es de suma importancia para la forma en que el analista se abre a las asociaciones del paciente y para la manera en que él mismo interviene en el proceso terapéutico. Si bien las diversas variantes de situaciones, reacciones y sentimientos contratransferenciales se pueden ordenar de forma relativamente fácil, vía investigaciones; la pregunta sobre la actitud que el terapeuta tiene que tomar lege artis, sobre este asunto, es más compleja.

\section{Freud: entre "abstinencia" y cercanía}

Si uno pidiera consejos a Freud llama la atención la contradicción entre la actitud relativamente estricta que él prescribe en sus escritos técnicos y la actitud que en concreto muestra cuando uno lo "ve" trabajar en sus casos. En sus escritos la actitud exhortada es muy clara: el analista tiene que detectar sus sentimientos lo más rápido posible y controlarlos (Freud, 1910, p. 108) ${ }^{3}$. En otro texto, acerca de la transferencia de enamoramiento de algunos pacientes, dice que el analista tiene que mantener una actitud

3. A.I. XI, p.136. 
"indiferente" optimiendo su contratransferencia (Freud, 1915, p. 313) ${ }^{4}$. Es a propósito de este tipo de relación que Freud subraya la importancia de un análisis propio, que más adelante se convertirá en una exigencia en la enseñanza del psicoanálisis. ${ }^{5}$

Freud no hace esta exhortación desde una moral de abstinencia puritana, tampoco desde un menosprecio hacia la autenticidad o valor intrínseco de los sentimientos del paciente y del terapeuta, por lo menos asi se desprende con claridad del contexto de su argumentación. Su preocupación mayor es el cuidado para el buen curso de la terapia, cuyo fin no puede perderse de vista. Cuando el analista reacciona a las pronunciadas necesidades afectivas del paciente con intereses que provienen de sus propios sentimientos personales, encierra al paciente dentro de las llamadas fronteras "seguras" de la terapia y le sustrae a éste la opción de una liberación obtenida por él mismo de las fijaciones perturbadoras de su vida sentimental. Entonces, y en ese sentido, oprimir la contratransferencia (que está exhortado) y atenerse a la abstinencia de satisfacción real dentro de la terapia (que está prescrito como regla) están inspirados por una motivación ética (deontológica). Los intereses personales (intereses amorosos) de la persona del terapeuta, así como su interés terapéutico por una cercanía, deben ceder radicalmente para que la abstinencia tenga preeminencia en la relación terapéutica que se ha iniciado con el paciente.

Con esta formulación de una de las reglas básicas del psicoanálisis es seguro que ningún psicoterapeuta tendrá algún problema, tampoco alguien de otra formación o escuela. La dificultad que posee esta formulación rígida de Freud, respecto a este "retiro", no está tanto en el fin que él propone sino en el medio que él prescribe al terapeuta: el dominio de la contratransferencia por represión de ella. Aparte de la pregunta de si esto es de alguna forma posible, existe la pregunta de si este medio

\footnotetext{
4. A.R. XII, p.168.

5. Iil autor se refiere al requisito del análisis didactico
} 
es la medicina apropiada para el fin propuesto. ¿Es la contratransferencia sólo un "diablillo" que tiene que ser oprimido?

Por supuesto que primero habría que hacer una pregunta aigo maliciosa, esto es, si el maestro se atenía a la prescripción hecha por él mismo. En estricto sentido, no. Si uno revisa las memorias que Freud escribió sobre algunos de los pacientes que él trataba con análisis, se ve trabajando a alguien con una libertad (sentimental) muy grande que a veces hasta entra en una discusión muy cercana con sus pacientes. Él no sólo escucha e interpreta, él también explica y discute. Además, pone mucha atención a una buena atmósfera de trabajo, una que ofrezca al paciente suficiente seguridad para atreverse y sostener la riesgosa empresa del análisis. Así, Freud es para el llamado bombre de las ratas como un amigo "que tranquiliza y mantiene derecho su sentimiento de autoestima". De esta manera él se preocupa del clima de confianza y seguridad que el paciente necesita para poder expresar más adelante sus sentimientos altamente hostiles (véase Stroeken, 1985, p. 97). Aparte de todo esto se sabe, por algunas publicaciones, que Freud a veces iba tan lejos en su apoyo a un paciente que no se asustaba de intervenir ayudando en la vida real. Recordemos la colecta que él promovió entre los analistas de Viena en favor del bombre de los lobos que había llegado desde Rusia completamente en bancarrota (véase Gardiner, 1971 y Obholzer, 1980).

No abordaremos otros detalles históricos. Algunos ejemplos son suficientes para mostrar el contraste entre prescripción y práctica concreta. Aparte del hecho de que los diversos casos de Freud, en las terapias efectuadas por él, nos enseñan algo sobre la personalidad del propio Freud, el contraste en cuestión nos enseña algo en general sobre el manejo del terapeuta respecto a sus sentimientos personales frente al paciente. Los ejemplos dados no serán entonces modelos de aplicación estricta de la regla escrita, es muy probable que en ellos la contratransferencia estuviera gobernada por el análisis pero, en todo caso, no estaba reprimida.

Ciertamente no se pueden leer las prescripciones técnicas separadas de los reportes sobre su aplicación concreta y, a la luz de la práctica, éstas 
parecen haber sido formuladas de manera demasiado estricta y unilateral. ¿Puede el terapeuta "simplemente" reprimir sus sentimientos? ¿Sería ésta la manera correcta de guiar el proceso terapéutico en buenas manos? ¿La "prescripción de represión" no sería acaso inconsecuente con el tono general de la manera analítica de trabajar? ¿Es la represión de los sentimientos una buena forma de dominarlos?

En la tradición psicoanalítica se han formado dos tendencias acerca de la cuestión de la contratransferencia, muy ligadas al contraste que se observa en el propio trabajo de Freud. Las que pueden ser denominadas, junto a Lane \& Storch (1986), cientifica clásica y bumanista. Ahondar más sobre esta divergencia nos ayudará a afinar las preguntas.

\section{La tendencia clásica}

En esta tendencia los autores ponen énfasis en la necesidad de dominar completamente la contratransferencia, esto significa reprimirla. Según ellos el terapeuta, conforme al consejo de Freud, "no debe ser transparente para el analizando, sino, como la luna de un espejo, mostrar sólo lo que le es mostrado" (1912b, p. 384) ${ }^{6}$. Este consejo se aplica estrictamente y guía la exigencia de que el analista no deje salir ni participar nada de sus sentimientos propios en el análisis. Él tiene que pasar desapercibido como persona real con características propias y con el menor contexto personal posible, preferentemente nada. En otras palabras, en su relación con el paciente, el analista tiene que mantener una neutralidad comparable tan sólo con aquella distante que imprime el observador científico a su estudio de los fenómenos de la naturaleza. En este contexto la interpretación es el único factor terapéutico activo que se conserva. Esta consistiría en dar una opinión racional, parca, basada en un análisis extento de cualquier elemento personal del analista, pero profunda en relación con todo lo que está pasando en la terapia. Tiene que ser dada lo más libre posible de valores, preferentemente en un lenguaje sin emociones. Estos

6. A.I. XII, p.117. 
autores hasta encuentran difícil que la neutralidad exigida en el análisis esté escrita como una neutralidad "benévola" (véase Dorpat, 1977). Ésta opinión está dada en forma enérgica por el analista entrevistado por Malcolm en su libro Psicoanálisis: una profesión imposible (1985): "La tarea del analista no es el manifestar simpatía al paciente, él tiene que darle entendimiento" (p. 86; véase también p. 122-123). En esta visión uno se une completamente a la metáfora en donde Freud llama al psicoanalista cirujano. En sus Consejos al médico, da el siguiente consejo: "No sé cómo encarecería bastante a mis colegas que en el tratamiento psicoanalítico tomen por modelo al cirujano que deja de lado todos sus afectos y aun su compasión humana, y concentra sus fuerzas espirituales en una meta única: realizar una operación lo más acorde posible a las reglas del arte" (Freud, 1912b, p. 380-381,7 véase también, entre otros, Freud, 1910, p. $110)^{8}$. Es verdad que muchas veces uno se olvida de seguir leyendo aquí para ver que Freud recomienda esta "frialdad sentimental" sobre todo para que el analista se guarde de temeridad terapéutica, y uno termina aplicando este consejo demasiado rápido al nacimiento y a la naturaleza misma de las interpretaciones. En todo caso es evidente que en ésta intención aséptica la contratransferencia será vista, mayormente, como un obstáculo, como un elemento estorbante para la terapia, como un quiebre en el espejo. Pareciera que la gran angustia que aquí participa tiene que ver de dos maneras con un superyó-imperativo terapéutico demasiado estricto. Uno no sólo tiene que preocuparse en demasia de que el ideal quirúrgico sea alcanzado lo más que se pueda en cada caso concreto, sino, que uno también tiene que cuidarse de la posibilidad de hacer de manera inesperada interpretaciones "contagiadas" (por sentimientos contratransferenciales) (véase Fine, 1986).

En esta tendencia es común que uno esté muy negativo frente a cada intento de cambiar la técnica analítica (véase Brenner, 1976, 1977) en la dirección de un mayor énfasis en la cercania real del terapeuta hacia el

\footnotetext{
7. A.I. XII, p. 114 .

8. A.li. XI, p. 136.
} 
analizado, en la empatía o en aquellos aspectos reales de la relación de trabajo. También que uno se sienta estremecido ante las intenciones de usar técnicas más activas en pacientes de patologia más severa, destinadas a dar un apoyo afectivo y a actuar durante el tiempo que sea necesario como un yo-apoyo para el yo-aportillado o frágil del paciente. En todo esto existe una preocupación ética de la mayor importancia acerca de la calidad del análisis y, según nosotros, nada tiene que ver con el descorazonamiento y la sequedad afectiva que la publicidad antianalítica se atreve a plantear. $\mathrm{El}$ interlocutor de Malcolm expresa muy bien el communis opinio de esta tendencia: "aunque parezca despiadado y autoritario, la neutralidad analítica estricta es una alternativa que da mucha más libertad; cuando disminuyes las durezas del análisis, con una gracia y amistad cuidadosamente dosificadas, quitas algo de la libertad del paciente porque tú decides qué es lo mejor para él” (Malcolm, 1985, p. 88).

Cuando se alinean las exigencias estrictas de los partidarios de la neutralidad radical, del distanciamiento inflexible y la pasividad en el análisis, surgen de manera inevitable suspiros de desesperación en aquellos que tienen que cumplir con esta tarea y también reacciones de desagrado en no-analistas que comparan el análisis con otras formas de psicoterapia. ¿No hablaba acaso Freud del psicoanálisis como una de las profesiones imposibles (aparte de la educación y la política)? Para él ésta no era una exclamación patética, vacía. En su correspondencia con Jung se ve claramente que Freud pensaba esto en relación a la represión según él necesaria de la contratransferencia. Jung, dando consejos en relación a una situación complicada de transferencia (y contratransferencia) en una de sus terapias, anuncia a Freud un poco irónico: "Ellas (tales experiencias) nos ayudan a desarrollar la piel gruesa que nosotros necesitamos y a dominar la 'contratransferencia', la que después de todo es un permanente problema para nosotros; ellas nos enseñan a desplazar nuestros propios afectos hacia una mejor ventaja" (McGuire, 1974, p. 231, carta F 145

9. "They (such experiences) help us to develop the thick skin we need and to dominate 'countertransference', which is after all a permanent problem for us; they teach us to displace our own affects to best advantage". 
del 7 de junio de 1909).

De éstas y otras observaciones parecidas uno tiene la impresión de que el análisis sólo es una profesión imposible y difícil, en la que uno tiene que mantener cualquier restricción afectiva porque es una actividad en la cual uno no puede, o mejor dicho no debe, experimentar placer. Según Fine (1986, p. 4-5), en todo esto la actitud de Freud es la "fuente del superyó analítico" que domina a tantos analistas.

Al igual que cuando prescribimos el valor e importancia de las actitudes analíticas fundamentales (neutralidad bien entendida, atención uniforme, no interferencia en la vida diaria del paciente y respeto máximo para su libertad creciente), las que están al servicio de la regla básica de la asociación libre, pensamos que hay que poner un gran signo de interrogación a la intención demasiado quirúrgica y aséptica que la tendencia clásica persigue. ¿Es la contratransferencia sólo un enemigo al que se tiene que temer para un buen curso del análisis? Si bien se puede estar de acuerdo con la necesidad de dominar la contratransferencia subsiste la pregunta de si su represión es el mejor camino para lograrlo. No se puede perder de vista el fin para el cual esta actitud terapéutica tiene que servir; es decir, para la promoción del hablar libre y liberado del paciente.

\section{La tendencia humanista}

La orientación "menos estricta" dentro del pensamiento analítico ha sido llamada por Lane y Storch (1986) la tendencia bumanista. Los autores de esta orientación encuentran apoyo para su actitud más "liberal" en la manera ya mencionada de tratar atrevida y, a veces, muy cercana de Freud a sus pacientes. Lipton (1977), por ejemplo, estudia de forma profunda la técnica y el estilo de relación de Freud en el tratamiento del bombre de las ratas y contrasta estos aspectos con lo que después se llamaría la técnica clásica que discutimos en la parte anterior. Llega a la conclusión de que esta técnica, si bien recurre a la manera de trabajar de Freud, ha hecho una selección de la empresa llevada a cabo por éste y en el fondo se trataría de técnicas diferentes. Cuando uno aplica los estándares estrictos de 
aquellos analistas que se llaman a sí mismos freudianos clásicos a la metodología que emerge de las terapias realizadas por Freud ésta, irónicamente, se derrumba. La diferencia, que los clásicos por lo demás no ven, está en el hecho que estos autores se "agarran" de una manera demasiado rigida, en la prescripción de la técnica, de aquellos textos en verdad técnicos en los que Freud recomienda que el analista tiene que ser como un espejo o tiene que trabajar como un cirujano, etc. Lo que los clásicos pierden de vista es que con todo ello Freud sólo hacía recomendaciones y no imponía prescripciones suficientes en si para un buen análisis. Las prescripciones se refieren al aspecto estrictamente técnico del análisis (hacer posible y promover asociaciones y crear buenas intervenciones) y no tienen la intención de abarcar y determinar toda la manera de ser e intervenir del analista. La necesidad de la neutralidad "se refiere a la operación misma y por supuesto no a cualquier otro contacto que existe necesariamente con el paciente" (Lipton, 1977, p. 272). La tendencia clásica ha dado a la idea "técnica" una extensión demasiado grande. Para Freud cada aspecto de la cooperación con el paciente no cae bajo las prescripciones técnicas. Sólo más tarde éstos serán extendidos a la relación personal entera que nace entre el analista y su paciente, una relación que por definición es individual e idiosincrática y que tiene que ser entendida desde su objetivo (admitir la perseverancia del análisis) y no desde un código rígido de orientación conductual e inmediatamente criticable (Lipton, 1977 , p. 272 ; véase también Mannoni, 1982, sobre todo p. 102 y siguientes).

Si uno pone demasiado énfasis en la prescripción freudiana de que el análisis tiene que transcurrir como un proceso quirúrgico aséptico, se concluirá que la tendencia clásica no considera un importante texto de los escritos técnicos de Freud. En Sobre la dinámica de la transferencia se hace una clara diferencia entre un aspecto de ésta que sólo es una consecuencia de los conflictos neuróticos reprimidos y otros componentes de la misma que se hacen más fácilmente conscientes y no despiertan resistencia: los sentimientos amistosos y tiernos que subsisten y que son "en el psicoanálisis, al igual que en los otros métodos de tratamiento, el portador del 
éxito" (Freud, 1912a, p.371) ${ }^{10}$. Es claro que el primer aspecto (el de los conflictos) tiene que llegar a ser el objeto del análisis; también, que el otro aspecto (el de los sentimientos amistosos) es el motor y que, por supuesto, no tiene por qué ser bloqueado por rigidez alguna o temor de contagio emocional. Casi en las primeras exposiciones extensas sobre su método, es decir, en los Estudios sobre la bisteria, ${ }^{11}$ Freud pone a menudo énfasis en la respuesta contratransferencial a ser considerada para el éxito de la terapia. Subraya la importancia de suficiente simpatía para el paciente y la necesidad de tomar diferentes actitudes de escuchar vía metáforas: el analista como profesor, como representante de una opinión mundial más libre y más considerada, como confesor que puede expresar su participación y respeto. Pareciera que en este texto antiguo, como en sus casos posteriores, no es todavía prohibido "crear algo humano para el paciente" (Freud, 1895, p. 285; véase también p. 264).

Freud mismo luchó siempre con la buena dosis de estos dos elementos, actitud técnica estricta y relación personal. Fsto casi no se esconde en sus casos. En los estudios críticos sobre los análisis publicados de Freud tampoco se evita esta cuestión (por ejemplo, Gardiner, 1971, sobre el "hombre de los lobos"). Es sobre todo en el período inicial de su actividad como analista que Freud era a veces demasiado intervencionista o ponía a sus pacientes bajo una presión interpretativa demasiado diligente, como desde un resorte dirigido a encontrar una afirmación de la "verdad" de sus descubrimientos.

Habria que decir sobre estos intentos contratransferenciales prejuiciosos que Freud no los tenía absolutamente a la vista ni desde el principio ni siempre, menos bajo control. Su actitud de conocimiento e interrogación rápida frente a Dora, es un ejemplo; queriendo descifrar contínuamente el secreto de sus relaciones escondidas la empujaba demasiado en una sola dirección: todas las interpretaciones van en el sentido de la fijación al padre.

10. A.E. XII, p. 103.

11. Iil antor se refiere al Apartado IV que fue elaborado por Freud, sin la colaboración de J. Breuer. 
Esta presión demasiado grande o el desconocimiento de otras líneas de interpretación, condujeron a la demolición prematura de la terapia (véase Corveleyn, 1984). En su estudio sobre Freudy sus pacientes Stroeken (1985) muestra la tendencia general de éste, más o menos sistemática y para todos los casos, de no considerar las referencias a la figura materna en el material analítico; así mismo, muestra que esta unilateralidad contratransferencial puede ser entendida desde la relación sólida y privilegiada pero muy reprimida con su madre. Es en estas experiencias concretas que Freud se dio cuenta de la importancia de la transferencia y contratransferencia en el análisis. Es de los errores y dificultades que él experimentaba en el trabajo analítico que él intentaba sacar las lecciones apropiadas. En sus escritos técnicos la repercusión de todo esto suena áspera, fría, severa y contiene sobre todo un sentido de aviso que evidencia la fuerte preocupación del propio Freud por la calidad del trabajo analítico en el grupo de alumnos, en rápido crecimiento. Quizás por eso llegó a ser un martillar demasiado ansioso sobre el mismo clavo pero, en todo caso y en otros contextos, nunca abandonó la idea sobre la importancia muy grande de la parte personal en la relación analítica (véase Freud, 1912a). La cercanía, la atención honesta y el respeto amistoso constituyen la fundación (contra)transferencial del trabajo terapéutico y no determinan que el trabajo de asociación y explicación ocurra de una manera personalmente interesada o afectivamente perturbadora (véase Mannoni; 1982).

Dentro del psicoanálisis son los autores de la tendencia humanista los que han puesto atención especial a los aspectos "reales" de la relación en la terapia; hablan por ejemplo de alianz̧a terapéutica, de coalición, de convenio básico terapéutico, etc. Este elemento propio de la relación analítica no tiene que ser puesto en el foco ni tiene que ser explicado (para afuera), si fuera así se sacaría el motor mismo del trabajo terapéutico. La actitud contratransferencial aquí implicada no es aquella de la neutralidad aséptica (¿con la indiferencia como "ideal"?) sino aquella de la atención benévola, personal. Para tematizar esto autores como Greenson (1960) y Kohut (1959) usan expresamente la noción de empatia de la terapia experiencial bumanista. Según ellos, la empatía es un elemento necesario para 
entender de manera suficiente al paciente y para crear una atmósfera suficientemente segura para la tarea exigente del análisis. Poland (1975) habla en este sentido de tacto como función analítica. Con esto no se refiere a una actitud de cortesía formal sino a una oficiosidad tangible para con el paciente, a un ser acompañado siempre en la búsqueda de la propia verdad. Esta actitud supone renuncias, mucho "retiro": el abandono de formas de pensar y de patrones de reacción, antes confiables y establecidos, y el desenmascaramiento de "cualquier casita sagrada y narcisista". Según Poland (1975) tacto se refiere a un modo de empleo sostenido del "conocimiento que está adquirido por un entendimiento cognitivo y por empatia" (p. 161). Se manifiesta en el buen timing y dosificación de las interpretaciones. Es el derivado técnico de la intención terapéutica del analista de promover el crecimiento personal del otro (véase también Fine, 1986, p. 14).

La tendencia humanista nos subraya una apreciación positiva de la contratransferencia. Su énfasis puesto en la importancia de una cercanía suficientemente positiva y real del analista, en su alianza con el paciente, nos muestra que los terapeutas no tienen por qué reaccionar de manera fóbica ni ante sus propios sentimientos personales ni ante la cercanía con el paciente. La contratransferencia ya no es tabú, tampoco un elemento incómodo que requiera represión, se constituye más bien en un componente aceptable y positivo de la relación terapéutica en general. Nosotros, sin embargo, pensamos que con esta aproximación más positiva no se ha dicho lo suficiente.

\section{La contratransferencia como elemento de elaboración de la verdadera interpretación}

Mientras la tendencia clásica considera la contratransferencia como un obstáculo para el análisis y la quisiera ver idealmente reprimida, la tendencia humanista la describe como un poder positivo, como un adjuvans con y como marco afectivo para el trabajo analítico estricto. ¿Esta aproximación positiva es suficientemente buena para las diversas posibilidades activas 
que el analista dispone como persona? ¿Es suficiente concederle un lugar valioso pero sólo a una distancia segura del trabajo "verdaderamente" analítico? ¿Si ella ya no es una plaga para el análisis, es entonces sólo un elemento delimitante de aquello que es fundamental para el análisis: la relación real que hace posible el trabajo terapéutico? Nosotros somos de la opinión que la contratransferencia al mismo tiempo forma una parte integral del llamado trabajo analítico estricto. En otras palabras no sólo es un fenómeno vincular delimitado, es también un ingrediente orgánico de la misma actividad interpretativa del analista. Un instrumento entonces. Con Theodor Reik (1935) se puede decir que el analista durante la sesión terapéutica no sólo tiene que tener una atención abierta y sin prejuicios para todo lo que mencione el paciente, también tiene que dirigir su escucha a sus propias asociaciones cambiantes que reflejan sus propias reacciones inconscientes (contratransferencia) a lo dicho. Sólo de esta manera se puede esquivar el arrecife de la explicación racional pura en el trabajo de interpretación, que de manera amenazante parece estar más en función de una construcción conceptual que ser el resultado de un escuchar activo al paciente en su particularidad afectiva. Un ejemplo extraordinario de este uso activo de la contratransferencia en la construcción de la interpretación está dado por el famoso analista de la escuela notteamericana Harold Searles $(1965,1979)$. Sus opiniones en su mayor parte están basadas en su trabajo terapéutico con pacientes esquizofrénicos, lo que no es un impedimento para nombratlo aquí como ilustración. Su visión de la contratransferencia como instrumento de la terapia es igualmente válida en otros dominios de la psicopatología, puesto que las exigencias a las que se somete el terapeuta en la terapia con pacientes esquizofrénicos sólo son como agrandamientos de las que suceden en toda terapia.

Durante quince años Searles trabajó en orientación psicoanalítica, y a tiempo completo, con pacientes esquizofrénicos en Chestnut Lodge (EE.UU). En esta "escuela", muy dura, este analista de formación ortodoxa aprendió las reglas básicas de la aplicación blanda y creativa del psicoanálisis en pacientes que no eran ayudados por las explicaciones clásicas-neutrales de transferencia y contenido de sus conflictos. Esto le fue posible por una (con)-memoración de la manera clásica del manejo de 
la contratransferencia. Con esta actitud, marcada por la neutralidad benévola y una disposición más bien pasiva-invitadora, la construcción de una relación sólida con el paciente esquizofrénico muchas veces ya caminaba mal. Inclusive, para entender la psicodinámica de estos pacientes, tampoco se podía llegar de manera suficiente a los elementos inmediatamente sensibles de sus asociaciones.

Para exponer algunas ideas de Searles sobre la contratransferencia nos introduciremos muy brevemente, sin detenernos en la terminología técnica, en su visión sobre el curso de la terapia en el paciente esquizofrénico. No es una vuelta inútil por un dominio tan especializado. Cada clínico sabrá reconocer la pertinencia de la opinión que "el trabajo con los pacientes más patológicos es útil en el aprendizaje de entender los conflictos de los menos patológicos, como también, al revés, el trabajo con pacientes menos patológicos ayuda a entender a pacientes mucho más patológicos... que se expresan de manera más dificultosa" (Searles, 1979, p.11).

La terapia con el paciente esquizofrénico comienza con una fase de transferencia simbiótica. Para llegar a una individuación saludable el paciente tiene que recibir la posibilidad de pasar por una fase de simbiosis terapéutica. En el principio la situación de transferencia es la simbiosis patológica en la cual el paciente se quedó fijado desde la más tierna infancia. Esta simbiosis es muy ambivalente: sentimientos de amor son mezclados con sentimientos más primitivos de odio, celos y venganza. Para entender esta fijación Searles no recurre a la hipótesis según él demasiado unilateral de la madre esquizofrenógena, él construye una hipótesis original de trabajo. En la confrontación con una figura materna que desde su propia patología sólo dispone de un yo incompleto, que por tanto no puede ser un objeto total, una buena madre para el futuro paciente, emerge en éste una tendencia básica que todos los niños bumanos tienen en común (querer llenar lo incompleto) y entonces él se propone como un sostén para los lugares débiles de aquel importante otro (la figura materna). La patología nace porque, al final, él no tiene éxito en esta empresa "terapéutica". Searles (1974), dice: "él no tuvo éxito en ayudar 
a su madre a llegar a ser para él un objeto materno total que se realiza en el rol de madre, la cual tenía un yo fragmentado" (p. 75). El sentimiento de culpa, que es la consecuencia de todo esto, llega a tener proporciones enormes y le persigue toda su vida. La patología autista se puede entender como una defensa necesaria contra este sentimiento de culpa terriblemente atormentador. En cierto sentido se puede decir que el paciente mantiene, durante toda su vida, su yo virtual al servicio de una eventual completación que cure a la figura materna. Cuando uno considera el yo-incompleto del esquizofrénico en su significación dinámica, y no como un rasgo estadístico, se puede decir que este defecto es en verdad el núcleo más vivo de su existencia (véase Searles, 1974, p. 27). Con esta intención de ayuda, él sale al encuentro del analista. En la transferencia el paciente retoma su iniciativa terapéutica que en aquél entonces falló en su intento de sostener al otro, de quien por lo demás él espera mucho en sus debilidades. Cuando el terapeuta oficial no toma esta iniciativa seriamente, por ejemplo, porque se agarra de ansiedad (por el reconocimiento de sus propias debilidades) y entonces de narcisismo (vinculado a su estatus oficial de terapeuta), el paciente no encuentra de ninguna manera una base suficientemente segura para empezar el proceso de individuación.

Ningún analista negará que las debilidades (restos de los estados de fijación primitivos y rasgos caracterológicos) también existen en el analista. Pero muchas veces, espontáneamente, sólo se acepta que son debilidades incómodas sólo para la terapia y que tienen entonces que ser radicalmente reprimidas. En el tratamiento de pacientes psicóticos las debilidades propias siempre son reveladas de forma inevitable. Como lo señala Searles la ansiedad ante esta revelación es una mala guía, mientras que el reconocimiento honesto nos enseña a entender más del paciente que la represión defensiva. Con demasiado gusto ( $y$, entonces, con excesiva defensa) el analista se agarra de la idea deseada de que él, con su análisis propio, ha podido solucionar todos sus problemas personales y que puede entonces estar siempre como alguien completamente sano y al servicio del paciente débil (Searles, 1974, p.71). Esta ilusión, que suena terapéutica pero que es narcicista, está reforzada a veces por exigencias inconscientes del superyó. Describiéndose a sí mismo como un analista recién iniciado da como 172 
ejemplo de esto "la voluntad para ayudar al paciente siempre, para darle siempre una atención constante y para mostrarle un interés ininterrumpido" (Searles, 1974, p. 252) y, también, "el esfuerzo de nunca experimentar un afecto negativo (!), menos expresarlo" (Searles 1974, p. 251). No son las debilidades o los rasgos personales del analista los que estorban en sí mismos el proceso terapéutico, es el no-reconocimiento de ellos. Puesto que hacen al analista sordo para los procesos intrapsíquicos, como para la movilidad de los afectos primitivos muy amenazantes, por los cuales pasa el paciente.

Muchas veces, como cualquier persona, el analista reacciona en sus encuentros con el psicótico con ansiedad, confusión interna, desamparo, enojo o impaciencia. Según Searles uno puede sacar una lección equivocada de la formación cuando, en respuesta a esto, uno recae en la actitud analítica estricta de neutralidad rígida y cuando uno intenta defenderse de la amenaza con interpretaciones racionales, limpias. Ellas no sirven, bloquean a los pacientes y esterilizan el proceso terapéutico en un vago status quo. De esta situación sólo será máximo el terapeuta quien saque una satisfacción inconsciente, es decit, el pensamiento que uno sí se inserta diligentemente mientras que el paciente se resiste al tratamiento (Searles, 1974, p. 72).

El proceso de individuación sólo puede comenzar cuando uno abandona la neutralidad defensiva y el impulso de interpretar según los patrones clásicos. Esta situación de transferencia y contratransferencia asimétrica tiene que ser elevada y esto sólo ocurre cuando el analista es capaz de reconocer completamente su inseguridad, su ansiedad y su desconocimiento (en cada terapia la misma siempre se inventa de nuevo) y puede, entonces, de esta forma aceptar al paciente en su persistencia de ayudar al otro (originalmente el miembro de la familia o el sistema incompleto). La debilidad, el ser incompleto del terapeuta, también es real y constituye el asidero real al que corresponde el "impulso terapéutico" del paciente, ya que éste no está exclusivamente cargado por proyecciones megalomaníacas (Searles, 1974, p. 72). Es sólo por este camino, opina Searles, que la transferencia originalmente caótica y muchas veces masiva, 
simbiótica, puede pasar a una simbiosis terapéutica. En esta situación de transferencia más simétrica e igualmente bien ordenada (reglas de timing, verbalización) el paciente recibe la oportunidad de adquirir, gracias al reconocimiento de su contribución positiva, una base suficientemente segura de un narcisismo "sano" que le puede servir más adelante como posición de salida para la individuación. La simbiosis terapéutica así prescrita es esencial y de la misma naturaleza que la simbiosis infantil normal, que estimula el crecimiento tanto del niño, como de la madre (Searles, 1974, p.74). Esta sólo puede comenzar cuando el terapeuta abandona su pequeño puesto de "único sano en la relación".

Searles no da muchas consideraciones generales sobre la manera de cómo esto puede realizarse de forma concreta; sin embargo, de sus diversos casos, nosotros extraemos las siguientes:

- Aceptar la tendencia de ayuda del paciente, lo que supone entre otras cosas ponerse menos activo en la interpretación de la transferencia, de la cual en la psicosis muchas veces sólo se puede entrever la conmoción.

- Dejar ser al paciente, en otras palabras no intentar entender inmediatamente su supuesto estado afectivo. Esto puede implicar que uno tenga que aprender tanto a tolerar un silencio dificil de tolerar, como que uno no puede escapar del mismo poniendo en palabras distanciadas las expresiones a veces dramáticas de ansiedad o las expresiones de desesperación interna que emergen del discurso delirante y caótico del paciente.

Aparte de abandonar el furor interpretativo, Searles también pone énfasis en la necesidad de abandonar la imperturbabilidad analitica exagerada y neutralidad absoluta. No se echa para atrás cuando se trata de tomar los propios sentimientos como punto de referencia para sus intervenciones, sobre todo, en momentos de estancamiento. Así dará expresión a veces a su enojo y a su impotencia, otras veces a su impaciencia o a su exigencia (egoísta) de respetar los límites importantes puestos por él. Esos sentimientos propios no sirven para elaborar una u otra construcción 
complicada; sin atribuir mucho, Searles toma sus propios sentimientos como base real para sus intentos de poner en palabras la situación de la relación. Enzarzando esos sentimientos en su intervención, él muestra a su paciente su debilidad y limitación humana y le aclara. De esta manera el paciente recibe un espacio para su motivación inconsciente de salvador. La no conmoción (adulteg) en cada posible situación afectiva sólo alimenta en el paciente la idea de generar odio, que el terapeuta no necesita nada de él, que él sólo está visto como un niño pequeño y dependiente.

Esta posición más simétrica de la contratransferencia permite la construcción del narcisismo del paciente en un espacio real. Además esta apertura mucho mayor frente a las emociones contratransferenciales da un instrumento importante al terapeuta. La posición más simétrica frente al paciente hace que el terapeuta pueda usar sus propias indicaciones afectivas como un medio de detección de la "naturaleza e intensidad de respuestas contratransferenciales y actitudes del paciente frente a él" (Searles, 1974, p. 252).

\section{Conclusiones}

Una cierta ortodoxia en el movimiento psicoanalítico se aferra al ideal quirúrgico del análisis; esto implica que la propia personalidad y reacciones del analista tienen que ser impedidas radicalmente como factores perturbadores de la terapia; y que la contratransferencia debe ser controlada y reprimida para llegar a la mejor neutralidad posible. La tendencia humanista, en cambio, señala que la contratransferencia no es a secas sólo un obstáculo para el análisis. Sin disminuir la exigencia de neutralidad, ponen énfasis en los aspectos externos y reales de relación analítica que apoyan sui idea de la terapia como una alianza de cooperación; al mismo tiempo, otorgan mucha importancia a la construcción de una cercanía suficiente y personal del analista como elemento que ayude a delimitar la misma labor analítica.

Nosotros opinamos que esta aproximación más positiva no es suficiente para estimar la real valía del rol e importancia de la contratransferencia. 
Esta no sólo se constituye en un elemento delimitante, implica también la totalidad de reacciones y representaciones afectivas que son evocadas en el analista en su confrontación con las asociaciones del analizado. Estas reacciones están intensamente relacionadas con el propio inconsciente y el analista ha aprendido a reconocerlas durante su análisis personal (análisis didáctico). Tales reacciones personales no se pueden dejar fuera de la contribución que pueden hacer a la labor analítica del paciente. En lugar de intentar reprimirlas de manera compulsiva en nombre de un ideal aséptico de neutralidad, estas reacciones pueden ser vistas como instrumento, como señales que muestran rastros para poder empezar con las explicaciones adecuadas. Este uso de la contratransferencia al servicio de la tarea terapéutica lo encontramos muy bien ilustrado en el trabajo clásico de Theodor Reik y, más recientemente, en el trabajo de Harold Searles sobre la terapia psicoanalítica en pacientes esquizofrénicos.

\section{Referencias}

Brenner, C. (1976). Psychoanalytic techniques and psychic conflict. Nueva York: University Press.

Brenner, C. (1977). Working alliance, therapeutic alliance, and transference. Journal of the American Psychoanalytic Association, 27 (supplement), 137157.

Corveleyn, J. (1984). Enkele beschouwingen omtrent de overdrachtproblematiek in het Dorageval van Freud. Psychoanalyse, 1, 31-37. Dorpat, T.H. (1977). On neutrality. Intermational Joumal of Psychoanalytic Psychotherapy, 6, 39-64.

Epstein, L. \& Feiner, A.H. , Eds. (1979) Countertransference. Nueva YorkLondres: Jason Aronson.

Fine, R. (1986). Countertransference and the pleasures of being an analyst. In H.J. Strean (Ed.), Countertransference:Current issues in psychoanalytic practice (Vol. 2, nrs. 3/4, pp. 3-19). Nueva York-Londres: The Haworth Press. 
Freud, S. (1895). Studien über Hysterie (Deel IV). G.W. I, 252-312. Eistudios sobre la Histeria (Parte IV) A.E. II, 261-309.

Freud, S. (1910). Die zukünftigen Chancen der psychoanalytischen Therapie. G.W. VIII, 103-115. Las perspectivas futuras de la terapia psicoanalitica. A.E. XI, 129-142.

Freud, S. (1912a). Zur Dynamik der Übertragung. G.W. VIII, 363-374. \{Sobre la dinámica de la transferencia. A.E. XII, 93-105\}

Freud, S. (1912b). Ratschläge für den Arzt bei der psychoanalytischen Behandlung. G.W. VIII, 357-387. Consejos al médico sobre el tratamiento psicoanalitico. A.E. XII, 107-119\}

Freud, S. (1915). Bemerkungen über die Übertragungsliebe. G.W. X, 305321. Puntualizaciones sobre el amor de transferencia (Nuevos consejos sobre la técnica del psicoanálisis, III) A.E. XII, 159-174\}

Gardnier, M. (1971). The Wolfman and Sigmund Freud. Londres: The Hogarth Press and the Institute of Psychoanalysis.

Greeson, R.R. (1960). Emphaty and its vicissitudes. International Joumal of Psychoanalysis, 41, 418-424.

Kohut, H. (1959). Introspection, empathy and psychoanalysis. Joumal of the American Psychoanalytic Association, 7, 4-59.

Lane,R.C. \& Storch, R.S. (1986). A fortuitus extra-analytic event: Counetransference, hidrance or benefit? En: H. S. Strean (Ed.), Countertransference: Current issues in psychoanalytic practice (vol. 2, nrs 3/ 4, pp. 33-43). Nueva York- Londres: The Haworth Press.

Lipton, S.D. (1977). The advantages of Freud's technique as shown in his analysis of the Rat Man. International Journal of Psychoanalysis, 58, 255-273.

Malcolm, J. (1985). Psychoanalyse: E en onmogelijk vak. Amsterdam: Wereldbibliotheek.

Mannoni, O. (1982). Ca n'empếche pas d'exister. París: Editions du Seuil. Mcguire, W. (Ed.) (1974). The Freud/Jung letters. The correspondence between Sigmund Freud and C.G. Jung. Londres: The Hogarth Press \& Routledge \& Keagan Paul.

Obholzer, K. (1980). Gespräche mit dem Wolfsmann: Eine Psychoanalyse und die Folgen. Reinbeck b/H: Rowohlt. 
Poland, W.S. (1975). Tact as a psychoanalytic function. Intermational Joumal of Psychoanalysis, 56, 155- 162.

Reik, T. (1935). Der überraschte Psychologe. ÜberËrraten und Verstehen unbewusster Voryänge. Leiden: A.W. Sijthoff's Uitgeversmaatschappii.

Searles, H. (1966). Collected papers on schizopbrenia and related subjects. Nueva York: International Universities Press. Escritos sobre esquizofrenia. Barcelona: Gedisa, 1980.

Searles, H. (1979). Countertransference and related subjects. Nueva York: International Universities Press. Wij citeren uit de beknoptere Franse versie: Le contre-transfert. París: Gallimard, 1981.

Sternbach, O. (1986). The cognitive problems of psychoanalysis or is psychoanalysis possible? En H. S. Strean (Ed.), Countertransference: Current issues in psychoanalytic practice (Vol. 2, nrs. 3/ 4). Nueva YorkLondres: The Haworth Press.

Strean, H. S. (Ed.) (1986).Countertransference: Current issues in psycboanalityc practive (Vol. 2, nrs. 3/4). Nueva York- Londres: The Haworth Press. Stroeken, H. (1985). Frend en zijn patiënten. Ter Elfder Ure, s.l.

Wolstein, B. (Ed.) (1988). Essential papers on countertransference. Nueva York: Nueva York University Press. 